\title{
THE PÓLYA-TCHEBOTARÖV PROBLEM
}

\author{
JOAQUIM ORTEGA-CERDÀ AND BHARTI PRIDHNANI
}

\begin{abstract}
We describe the solutions to the problem of identifying the continuum in the complex plane that minimizes the logarithmic capacity among all the continuum that contain a prefixed finite set of points. This description can be implemented numerically and this can be used to improve the estimates on the Bloch-Landau constant and other related problems as the maximal expected lifetime of the Brownian motion on domains of inner radius one or the principal eigenvalue for the Laplace operator on such domains.
\end{abstract}

\section{INTRODUCTION AND HISTORY OF THE PROBLEM}

Pólya in Pól29 discussed the following problem which was suggested to him by Tchebotaröv:

Problem 1. Given a finite number of points $E:=\left\{a_{1}, \ldots, a_{n}\right\} \subset \mathbb{C}$, find the continuum $K$ with minimal logarithmic capacity such that $E \subset K$.

For any continuum $K$, its complement in the Riemann Sphere, $\Omega=\mathbb{C}_{\infty}$ is simply connected, therefore there exists a unique conformal map $f: \Omega \rightarrow \mathbb{C}_{\infty} \backslash D(0, R)$ such that $f(z)=z+c_{0}+c_{-1} / z+\cdots$ around $\infty$. Here $R=R(\Omega, \infty)$ is called the conformal radius of $\Omega$ with respect to $\infty$. In fact, $\operatorname{cap}(K)=R(\Omega, \infty)$. This provides an equivalent formulation of Problem 1 which is usually called the outer reformulation of the Pólya-Tchebotaröv problem:

Problem 2. Given a finite number of points $E:=\left\{a_{1}, \ldots, a_{n}\right\} \subset \mathbb{C} \backslash\{0\}$ find a conformal map $f: \mathbb{D} \rightarrow \mathbb{C} \backslash E$ such that $f(0)=0$ and $\left|f^{\prime}(0)\right|$ is maximal.

So we are looking for a simply connected domain $\Omega$ that contains the origin, it is contained in $\mathbb{C} \backslash E$ and such that the density of the hyperbolic metric at the origin is minimal. Such domain will be called an extremal domain and the corresponding conformal map, an extremal map.

The existence of the solution is obvious by a normal family argument. This problem was studied in detail by Laurentiev in Lau30. He proved the uniqueness and the basic structure of the solution by the method of variations of the boundary. The structure of the extremal domain is characterized by the following theorem, see Lau34.

Theorem 1 (Laurentiev). Given a finite number of points $E=\left\{a_{1}, \ldots, a_{n}\right\} \subset$ $\mathbb{C}$, there exists a unique extremal domain $\Omega=f(\mathbb{D})$ for the problem 2 and it is characterized by the following properties:

(1) Each point of the plane belongs to either $\Omega$ or $\Gamma:=\partial \Omega$.

Date: August 23, 2021.

2000 Mathematics Subject Classification. Primary .

Supported by DGICYT grant MTM2008-05561-C02-01 and the CIRIT grant 2005SGR00611. 
(2) The boundary $\Gamma$ consists of finitely many simple arcs of analytic curves. The points $a_{i}$ and $\{\infty\}$ are endpoints of $n+1$ distinct arcs. Every point of $\Gamma$ different from the $a_{i}$ or $\{\infty\}$ either belongs to a unique arc and it is a regular point of $\Gamma$, or it is the common end of at least three arcs.

(3) To any arc $\alpha \beta$ consisting of regular points of $\Gamma$ there correspond under the conformal mapping $f^{-1}$ two arcs of the same length on the unit circle.

When the last property 3 holds we say that the arcs are harmonically symmetric with respect to the origin and it will be the key property to find a numerical algorithm to determine the solution to the problem mentioned above.

In the proof of this last theorem, Laurentiev assumed that the desired domain is bounded by finitely many simple Jordan arcs. This assumption was removed by Goluzin who used the method of inner variations to prove the following:

Theorem 2 (Goluzin, Gol46). Let $a_{1}, \ldots, a_{n}$ be arbitrary given points in $\mathbb{C}$. Let $K$ be the extremal continuum for Problem 1 . Then $K$ is the union of the closures of all critical trajectories of the quadratic differential

$$
Q(z) d z^{2}=-\frac{\prod_{l=1}^{n-2}\left(z-b_{l}\right)}{\prod_{k=1}^{n}\left(z-a_{k}\right)} d z^{2}
$$

where $b_{l}$ are some unknown parameters. The extremal univalent function $g: \mathbb{C}_{\infty} \backslash$ $\mathbb{D} \rightarrow \mathbb{C}_{\infty} \backslash\left\{a_{1}, \ldots, a_{n}\right\}$, with $g(\infty)=\infty$ that maximizes $g^{\prime}(\infty)$ must satisfy the following differential equation

$$
\left(z g^{\prime}(z)\right)^{2}=\frac{\prod_{i=1}^{n}\left(g(z)-a_{i}\right)}{\prod_{j=1}^{n-2}\left(g(z)-b_{j}\right)} .
$$

Remark 1 . The points $b_{i}$ correspond to common end points of several arcs. If some point $b_{i}$ is a common end of $m$ arcs, then the term $g(z)-b_{i}$ will appear exactly $m-2$ times in the differential equation.

Later we will explain how to use this differential equation to obtain a numerical solution to Problem 2,

Goluzin gave a more general result where the problem is to maximize $\left|f^{(n)}(0)\right|$ for any $n \geq 1$. An account of his work is in Gol69, Chap. 4]. By using this description and after considerable work, Kuzmina in Kuz82 computes the extremal domain in the case of three points and in Fed84 this is extended to four points with a certain symmetry (two of the points must be symmetric with respect to a line that passes by the other two).

Later on, Tamrazov found an explicit solution for the problem of $n$ points. The general solution to Problem 2 is, according to Tam05, of the form

$$
f(z)=\int^{z}(\zeta-1)^{-3}\left(\prod_{\alpha \in V \backslash\{1\}}(\zeta-\alpha)\right) \prod_{v \in W(\Gamma)}\left(\prod_{\beta \in W_{v}}(\zeta-\beta)\right)^{1 / \tau(v)} d \zeta,
$$

where $\alpha$ and $\beta$ are a finite number of points in $\mathbb{T}$ and $\tau(v)$ is a positive integer. This result does not seem to be completely clear, because the function $f$ corresponds to a Schwartz-Christoffel formula, thus $f(\mathbb{T})$ is going to be a collection of straight segments (one of them going to $\infty$ ) but, even when we have only three points, in most cases (except of very symmetric ones) the solution to the Pólya-Tchebotaröv problem are not straight lines. 
Nevertheless the main idea of Tamrazov paper, that all solutions can be exactly parametrized by a finite planar graph is indeed correct. We will give a different proof of this fact. Our approach although it will not yield an "explicit" formula it will be constructive and it is possible to implement a numerical algorithm that produces an approximation to the solution of the Pólya-Tchebotaröv problem.

On Sections 2 and 3 we prove that all solutions are codified by a "nested partition" which are defined there. This is more convenient for us, although it is completely equivalent to a parametrization by graphs. To each set of points the extremal continuum is in correspondence with a unique "nested partition" of $\mathbb{T}$ and conversely, each "nested partition" provides a solution. Thus all the combinatorial data of the solution is codified in these partitions.

Once we have a parametrization of all possible solutions we introduce in 4 a numerical algorithm to compute numerically the solutions (i.e. to determine the parameters) to the Pólya-Tchebotaröv problem. We illustrate the method making it explicit in the case of 3 points and 6 points (with a certain symmetry). This last case is particularly interesting because it will be of use for the applications that we had in mind which are developed in Section 5

The Pólya-Tchebotaröv problem is rather basic, thus it is not surprising that it arises in connection with many other problems. The most evident case is in the estimates of the univalent Bloch-Landau constant, the precise formulation of the problem is in Section 5. This has been exploited in [COC08 where this constant was improved. This work is its natural continuation. Here we will provide more sophisticated examples and we will use the same type of domains to improve the estimates of two other extremal problems that were introduced in [BC94]: the expected lifetime of the Brownian motion in a domain with inner radius one and the estimation of the principal frequency of such domains. For the precise definitions and results, see again Section 5 .

There are other potential applications of the Pólya-Tchebotaröv problem which could benefit from our (numerical) solution. For instance the best estimates in the Smale mean value conjecture obtained in Cra07 rely on the computation of the solution of the problem with three points. We have not pursued improvements on this problem.

Acknowledgment: We are indebted to Àlex Haro for illuminating conversations about the numerical implementation of the algorithm.

\section{The PARAMEtrization of AlL SOlUtions}

In view of Laurentiev and Goluzin results, the continuum that is a solution of Problem 1 form a finite planar tree with endpoints in the points $\left\{z_{1}, \ldots, z_{n}\right\}$. The remaining nodes are of order at least three. This motivates the following definition introduced in [Tam05:

Definition 1. A graph $\Gamma$ is a Pólya-Tchebotaröv graph (PT-graph for short) if it is a finite planar tree with the properties:

(1) All sides of the graph are linear segments.

(2) There are no nodes of order 2.

(3) The sum of the length of all sides is exactly $1 / 2$.

We say that $\Gamma$ is normalized if we mark one of the vertex of the tree (a node of order one). Two PT-graphs are equivalent if there is an isometry from one to 
the other that extends to an orientable homeomorphism of the plane. If they are normalized we also require that the isometry sends the marked vertex from the first graph to the marked vertex of the second graph. We will talk of a PT-graph $\Gamma$ to denote the whole equivalence class.

Since Problem 1 is invariant by translation we can be normalize the data $\left\{z_{1}, \ldots, z_{n}\right\}$ to assume that $z_{1}=0$.

The main result is that there is a natural way of parametrizing the solutions to the Pólya-Tchebotaröv problem by normalized PT-graphs. That is for any graph there is associated a unique continuum that solves a Pólya-Tchebotaröv problem and conversely all solutions arise in this way.

Let us describe how to associate a solution to each graph. First we need one further definition.

Definition 2. A partition $\Pi$ of the unit circle in a finite number of intervals is called a properly nested partition if the following properties hold:

(1) The intervals come in pairs of equal length, i.e.

$$
\Pi=\left\{I_{1}, \ldots, I_{n}\right\} \cup\left\{J_{1}, \ldots, J_{n}\right\}
$$

and $\left|J_{i}\right|=\left|I_{i}\right|$ for all $i=1, \ldots, n$.

(2) There are no nested pairs of intervals, i.e. a couple $I_{i}, J_{i}$ never separates another couple $I_{k}, J_{k}$. See Figure 1
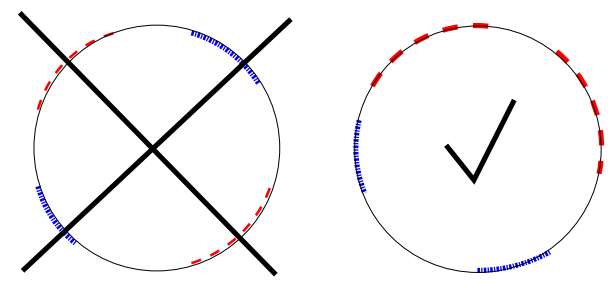

Figure 1. Not nested and nested pairs

Since all pairs are not nested we can be sure that there exist at least one pair $I_{i}, J_{i}$ of adjacent intervals. Two partitions are equivalent if one rotation sends one to the other. They are normalized if we mark one of the adjacent pairs of intervals.

It is easy to see that any PT-graph provides a nested partition and conversely. We start from a vertex of the graph and travel through its edges directwise. To each edge of the tree we associate an interval in the circle of the same length (on the unit circle we consider the normalized length). Each edge of the tree is visited twice, once for every side. We consider the pairs of intervals $I_{i}, J_{i}$ to intervals that correspond to different sides of the same edge of the tree.

Finally for any given properly nested partition there is associated an involution $\tau$ defined on the circle (except in a finite number of points corresponding to the end points of the intervals). Two points $x, y$ are related by the involution $\tau$ if $x$ belongs to the interval $I_{i}$ and $y$ belongs to its pair $J_{i}$. The definition of $\tau$ in each of the intervals is the reflection along the diameter of the disk that passes halfway in between the pair of intervals as in Figure 2

We are going to prove a "welding" type theorem: 


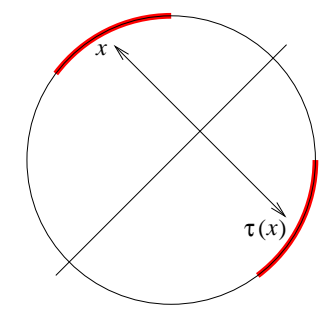

FIGURE 2. The involution $\tau$ restricted to a pair of intervals

Theorem 3. For any given properly nested partition $\Pi$ of $\mathbb{T}$ and its associated involution $\tau$ there is a conformal map $f_{\Pi}: \mathbb{D} \rightarrow \mathbb{C} \backslash \Gamma$ where $\Gamma$ is a finite union of analytic arcs $\Gamma=\cup_{i=1}^{n} \gamma_{i}$ such that the image of any pair $I_{k}, J_{k} \subset \Pi$ is one arc $\gamma_{k}$ and $f_{\Pi}(x)=f_{\Pi}(\tau(x))$ for all $x$ in the intervals. Moreover $f_{\Pi}$ is unique up to postcomposition with automorphisms of $\mathbb{C}$.

Remark 2. If we compose $f_{\Pi}$ with a translation we obtain a function that satisfies the Laurentiev conditions of Theorem 1. Thus for any $\Pi$ we get a solution to Problem 2. The converse is even more clear. Laurentiev theorem shows that the boundary of the extremal domain $\partial \Omega$ is a tree, that is homeomorphic to a rectilinear PT-graph $\Gamma$. The length of each edge of $\Gamma$ is one half of the harmonic measure of the corresponding edge of $\partial \Omega$. The conformal map $g: \Omega \rightarrow D$ with $g(0)=0$ gives a partition $\Pi$ of the unit circle. By property (3) of Laurentiev theorem, we get $g^{-1}=f_{\Pi}$.

\section{Proof of the Welding Theorem}

Given the partition we will proceed to construct the mapping in a finite number of steps. In each step the following lemma is the key

Lemma 1. Given two adjacent intervals $I, J \subset \mathbb{T}$ in the circle, such that $\mathbb{T} \neq$ $\{I \cup J\}$ and a quasisymmetric homeomorphism $\tau: I \rightarrow J$ that fixes the common point $p$, there is a simple Jordan arc $\gamma \subset \mathbb{D}$ with one endpoint at 1 and a conformal mapping $f: \mathbb{D} \rightarrow \mathbb{D} \backslash \gamma$ such that $f(0)=0$ and $f(x)=f(\tau(x)) \in \gamma$ for all $x \in I$. The mapping $f$ and the curve $\gamma$ are unique, they depend on $I, J$ and $\tau$.

Proof. Let $I_{0}=\left\{e^{i t} ; t \in[0, \pi / 2]\right\}$ and $J_{0}=\left\{e^{i t} ; t \in[-\pi / 2,0]\right\}$, and let $\tau_{0}: I_{0} \rightarrow J_{0}$ be defined as $\tau_{0}(z)=\bar{z}$. If these were the data of the problem, it will be readily solved by the mapping $f_{0}(z)=\ldots$ that maps $\mathbb{D}$ to $\mathbb{D} \backslash[r, 1)$. In the general situation, there exists an homeomorphism of the circle $T: \mathbb{T} \rightarrow \mathbb{T}$ such that $T(I)=I_{0}$, $T(J)=J_{0}$ and $\tau_{0}(T(x))=T(\tau(x))$ for all $x \in I$. In $I$, the map $T$ is defined linearly. On $J$ we use as definition $T(y)=\tau_{0}\left(T\left(\tau^{-1}(y)\right)\right)$ and outside $I$ and $J$ we define it linearly. Since $\tau$ is asymmetric then $T$ is quasisymmetric. In general $T$ has the same regularity as $\tau$. By the Beurling-Ahlfors extension theorem BA56] there is a quasiconformal homeomorphism of the disk that extends $T$. We will still denote it by $T$.

Let $g=f_{0} \circ T$. The map $g$ is mapping $I$ and $J$ to the $\operatorname{arc}[r, 1]$ in such a way that $g(x)=g(\tau(x))$ for all $x \in I$. It is not a conformal, but it is a quasiconformal map because $T$ is quasiconformal and $f_{0}$ is conformal. This can be corrected by solving a Laplace-Beltrami equation. We want an homeomorphism $\Psi: \mathbb{D} \rightarrow \mathbb{D}$ such that 
$k=\Psi \circ g$ is conformal. Thus $k_{\bar{z}}=\Psi_{\bar{z}} \overline{g_{z}}+\Psi_{z} g_{\bar{z}}=0$. There is always a solution $\Psi$ to this Laplace-Beltrami equation that is an homeomorphism from $\mathbb{D}$ to $\mathbb{D}$ since $\left|g_{\bar{z}} / g_{z}\right|<k<1$ almost everywhere by the measurable Riemann mapping theorem. Finally we compose $k$ with an automorphism of the disk $\kappa$ and the desired function is $f=\kappa \circ k$. The automorphism $\kappa$ is chosen to make sure that $f(0)=0$ and the endpoint of $f(I)=\Psi([r, 1])$ is at 1 . The regularity of $f$ at the boundary is as good as that of $\Psi$ and that itself is determined by the regularity of $\tau$.

If there were two curves $\gamma_{0}$ and $\gamma_{1}$ and two maps $f_{0}$ and $f_{2}$ with the same property then $g=f_{1} \circ f_{0}^{-1}$ is a conformal mapping from $\mathbb{D} \backslash \gamma_{0}$ to $\mathbb{D} \backslash \gamma_{1}$ such that $g(0)=0$. Moreover $g$ extends continuously to $\gamma_{0}$ because for any point in $\gamma_{0}$ the preimage by $f_{0}$ are two points $\{x, y\}$ in $\mathbb{T}$ that are related by $\tau$, thus $f_{1}(x)=f_{1}(y)$, therefore $g$ extends continuously to $\gamma_{0}$ and therefore it extends analytically, thus $g(z)=e^{i \theta} z$. Since both $\gamma_{0}$ and $\gamma_{1}$ start have an endpoint in 1 , then $g(z)=z$ and $f_{0}=f_{1}$.

With the same proof, mutatis mutandi, we deal with the case $\mathbb{T}=I \cup J$ and we obtain

Lemma 2. Given two adjacent intervals $I, J \subset \mathbb{T}$ in the circle, such that $\mathbb{T}=$ $\{I \cup J\}$ and a quasisymmetric homeomorphism $\tau: I \rightarrow J$ that fixes the common points, there is a simple Jordan arc $\gamma \subset \mathbb{C}$ with one endpoint at 1 and the other at $\infty$ and a conformal mapping $f: \mathbb{D} \rightarrow \mathbb{C} \backslash \gamma$ such that $f(0)=0, f^{\prime}(0)>0$ and $f(x)=f(\tau(x)) \in \gamma$ for all $x \in I$. The mapping $f$ and the curve $\gamma$ are unique, they depend on $I, J$ and $\tau$.

Proof of the theorem. We take any pair of adjacent intervals $\left(I_{k}, J_{k}\right)$ in the partition $\Pi$ corresponding by the involution $\tau$. There are always adjacent pairs because the partition is properly nested (they correspond to edges with and endpoint in a vertex of the graph). Applying the Lemma we find a conformal mapping $f_{1}$ that welds together the pair of intervals in a curve $\gamma_{1} \subset \mathbb{D}$. The mapping $f_{1}$ induces a new partion $\Pi_{1}$ of $\mathbb{T}, I_{j}^{1}=f_{1}\left(I_{j}\right)$ and $J_{j}^{1}=f_{1}\left(J_{j}\right)$ for all $j$ except for the pair $\left(I_{k}, J_{k}\right)$ that was welded together. This new partition is again correctly nested because the order is preserved except for a pair of adjacent intervals that "collapses". The number of pairs of intervals is one less than in $\Pi_{1}$. The intervals in each pair are no longer of the same size but nevertheless the map $f_{1}$ induces a new involution on them, $\tau_{1}=f_{1} \circ \tau \circ f_{1}^{(-1)}$.

Now we repeat the procedure. We take any other new pair of adjacent of intervals of the new partition $\Pi_{1}$ corresponding by $\tau_{1}$ and we glue them together. This can be done by a mapping $f_{2}$ applying the Lemma because $\tau_{1}$ is quasisymmetric (it is in fact piecewise real analytic). In this way we get again a new involution $\tau_{2}=f_{2} \circ \tau_{1} \circ f_{2}^{(-1)}$ and a new nested partition $\Pi_{2}$.

In this way we keep gluing pairs of intervals until we are left only with two intervals and an involution $\tau_{n}$ that relates them. In this last step we use Lemma 2 to get $f_{n}$. The final conformal mapping is $f_{\Pi}=f_{n} \circ \cdots \circ f_{1}$.

There is basically only one such map $f_{\Pi}$ (except for composition with maps of the form $a z+b)$. The proof is as in Lemma 1 suppose there is another such map $g_{\Pi}$. Let $\Gamma=f_{\Pi}(\mathbb{T})$, then $h=g_{\Pi} \circ f_{\Pi}^{-1}$ is a one to one analytic mapping $h: \mathbb{C} \backslash \Gamma \rightarrow \mathbb{C}$. Moreover since the preimage of any regular point in $\Gamma$ are two points in the circle that are related by the involution, and $g$ maps both points to 
the same point, then $h$ can be extended continuously to a conformal map from $\mathbb{C}$ to $\mathbb{C}$, thus $h(z)=a z+b$.

\section{Numerical Algorithm to find solutions}

As we mentioned above, in order to implement numerically an algorithm to find the solution to Problem 2 we used an important property of the extremal domain and the differential equation obtained by Laurentiev. Denote by $\Omega_{n}$ the desired extremal domain for the Problem 2 in case of $n+1$ points $\left\{a_{1}, \ldots, a_{n}, \infty\right\}$. Let $f: \mathbb{D} \rightarrow \Omega_{n}$ be the conformal map such that $f(0)=0$. We know that $f$ satisfies the following differential equation

$$
\left(\frac{z f^{\prime}(z)}{f(z)}\right)^{2}=C \frac{\prod_{i=1}^{n}\left(f(z)-a_{i}\right)}{\prod_{j=1}^{n-1}\left(f(z)-b_{j}\right)},
$$

where the parameters $b_{j}$ are unknown and $C=\frac{\prod_{l=1}^{n-1}\left(-b_{l}\right)}{\prod_{k=1}^{n}\left(-a_{k}\right)}$. Using the solutions of this differential equation and the last property of Theorem 1 we have implemented the resolution of Problem 2 for some cases of $n$. The system becomes more delicate as $n$ increases, the combinatorics and the dimensions of the systems to solve become bigger. We will show in detail the solution in the case $n=3$ to illustrate the method and $n=6$ with some extra symmetry, because this will be enough for the applications that we have in mind. The code where this algorithm is implemented (for four points and 6 points with symmetry) can be downloaded from http://www.maia.ub.es/cag/code/tchebotarev/.

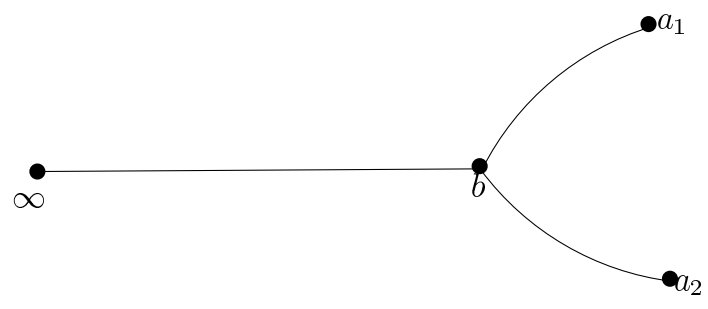

FIGURE 3. Sketch of the extremal compact for three points

4.1. Case of 3 points. Let's start with 3 points. Assume that we have $a_{1}, a_{2}, a_{3}$ three points such that $a_{3}=\infty$ and $a_{1}, a_{2} \neq 0$. Without loss of generality we will always assume that $f(1)=\infty$. In the case of three points the extremal domain is very clear (see Figure 3). We only have one unknown parameter denote it by $b$ in the differential equation (11) that reduces to:

$$
f^{\prime}(z)^{2}=C \frac{\left(f(z)-a_{1}\right)\left(f(z)-a_{2}\right)}{f(z)-b} \frac{f(z)^{2}}{z^{2}}
$$

Recall that to any regular arc $\alpha \beta$ of $\partial \Omega$ there corresponds two arcs with equal lengths on the unit circle. So this means that we have the configuration on the unit circle shown in Figure 4 , where $f(0)=0$, the arcs $\left(1 \beta_{1}\right),\left(\beta_{3} 1\right)$ are mapped to the $\operatorname{arc} \infty b, \beta_{1} \alpha_{1}$ and $\alpha_{1} \beta_{2}$ are mapped to the arc $b a_{1}$ and the $\operatorname{arcs} \beta_{2} \alpha_{2}, \alpha_{2} \beta_{3}$ into $b a_{2}$. Note that $f\left(e^{i \alpha_{i}}\right)=a_{i}$ for $i=1,2$ and $f\left(e^{i \beta_{i}}\right)=b$ for $i=1,2,3$. 


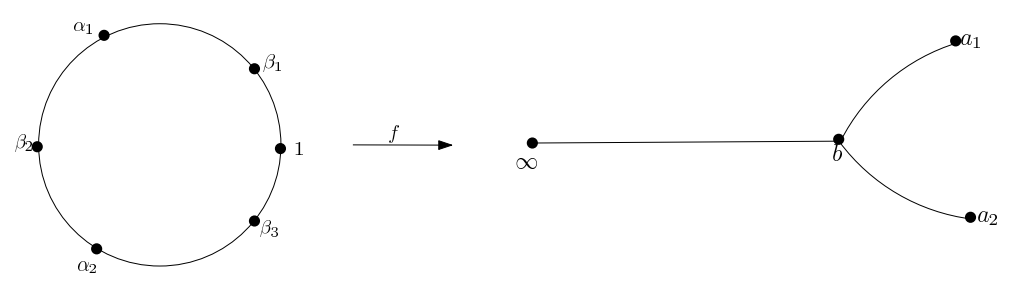

FIgURE 4. Configuration for $n=3$

The solution of the problem can be viewed as the solution of a system of nonlinear equations. If we know the value of $f^{\prime}(0)$ and $b$ we can compute the coefficients of the mapping $f$ using the differential equation (2). We computed also the values of $\alpha_{i}, \beta_{i}$. Note that as the arcs $1 \beta_{1}, \beta_{3} 1$ must have same length, we get $\beta_{3}=2 \pi-\beta_{1}$ (we will always take the angles in the range $[0,2 \pi)$ ). So, we have 6 real unknown parameters in our problem: $\operatorname{Re}\left(f^{\prime}(0)\right), \operatorname{Im}\left(f^{\prime}(0)\right), \operatorname{Re}(b), \operatorname{Im}(b), \beta_{1}, \beta_{2}$ and using the last property of Theorem 1 we can impose the following three complex equations

$$
\left\{\begin{array}{l}
f\left(e^{i \beta_{1} / 2}\right)=f\left(e^{-i \beta_{1} / 2}\right) . \\
f\left(e^{i\left(\alpha_{1}+\beta_{1}\right) / 2}\right)=f\left(e^{i\left(\alpha_{1}+\beta_{2}\right) / 2}\right) . \\
f\left(e^{i\left(\alpha_{2}+\beta_{2}\right) / 2}\right)=f\left(e^{i\left(\alpha_{2}+\beta_{3}\right) / 2}\right) .
\end{array}\right.
$$

We used a the hybrid method to find an approximation of the roots of the system (see Pow70 for more details of the method).

To apply the root-finding method we need to evaluate $f\left(e^{i \gamma}\right)$ for any $\gamma \in[0,2 \pi) \backslash$ $\left\{\alpha_{1}, \alpha_{2}, \beta_{1}, \beta_{2}, \beta_{3}\right\}$. For that, denote $z(t)=f\left(t e^{i \gamma}\right)$. We know that $z(0)=0$ and $z^{\prime}(0)=f^{\prime}(0) e^{i \alpha}$. Note that $z(1)=f\left(e^{i \alpha}\right)$. We can get the differential equation satisfied by $z(t)$ and solve it to obtain the value in time $t=1$. We get

$$
z^{\prime}(t)^{2}=C \frac{\left(z(t)-a_{1}\right)\left(z(t)-a_{2}\right)}{(z(t)-b)} \frac{z(t)^{2}}{t^{2}}
$$

Note that this equation only defines $z^{\prime}(t)$ up to a sign, we will deal with this problem by analytic continuation. Once we fix the derivative at the origin there is a single analytic branch that solves the equation. To solve it we used the Taylor integration method which allows us to integrate the singularity in $t=0$. As $f$ is conformal, we know that $z(t)=z_{1} t+z_{2} t^{2}+\ldots$, where $z_{1}=f^{\prime}(0) e^{i \gamma}$. Now if we do the calculations in the equation (3) we get a recurrence for the coefficients till the order we want. Now we can estimate the radius of convergence of the obtained series. And therefore proceed using Taylor method to integrate the differential equation until $t=1$. Hence we will be able to impose the equations to solve our problem.

4.2. Case of 6 points with symmetry. Now consider the case of 6 points $a_{1}, a_{2}, \ldots, a_{6}$ such that $a_{6}=\infty, a_{3} \in \mathbb{R}$ and $a_{5}=\overline{a_{1}}, a_{4}=\overline{a_{2}}$. The extremal compact in this case may be of two types (see Figures 5, 67and 7). So, we have two type of configurations in this special case

(1) $0 \beta_{1}^{1} \alpha_{1} \beta_{2}^{1} \beta_{1}^{2} \alpha_{2} \beta_{2}^{2} \alpha_{3} \beta_{3}^{2} \alpha_{4} \beta_{4}^{2} \beta_{3}^{1} \alpha_{5} \beta_{4}^{1} 2 \pi$

(2) $0 \beta_{1}^{1} \beta_{1}^{2} \alpha_{1} \beta_{2}^{2} \alpha_{2} \beta_{3}^{2} \beta_{2}^{1} \alpha_{3} \beta_{3}^{1} \beta_{1}^{3} \alpha_{4} \beta_{2}^{3} \alpha_{5} \beta_{3}^{3} \beta_{4}^{1} 2 \pi$,

where $f\left(e^{i \beta_{k}^{j}}\right)=b_{j}$ for $j=1,2$ and $k=1,2,3,4, f\left(e^{i \alpha_{i}}\right)=a_{i}$ for $i=1,2,3,4,5$ and $f(1)=\infty$. Using the symmetry we can do some reductions to get a system of equation with less dimension. For example, we can always assume that the point 

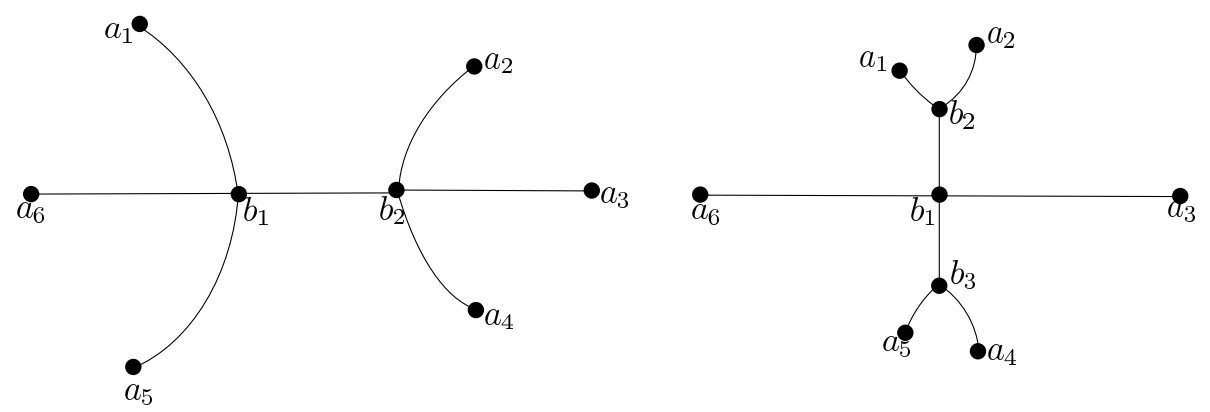

Figure 5. Structure of the extremal domains for $n=6$ with symmetry

$a_{3} \in \mathbb{R}$, so that $b_{1}, b_{2} \in \mathbb{R}$ for the first configuration and $b_{1} \in \mathbb{R}$ for the second one. Note that in this last configuration, by the symmetry of the problem, $b_{3}=\overline{b_{2}}$. Moreover as this is a symmetric case, $f^{\prime}(0)$ must be real and $\alpha_{3}=\pi$.

First configuration. In this case we have 7 real unknown parameters: $\operatorname{Re}\left(f^{\prime}(0)\right)$, $\operatorname{Re}\left(b_{1}\right), \operatorname{Re}\left(b_{2}\right), \beta_{1}^{1}, \beta_{2}^{1}, \beta_{1}^{2}, \beta_{1}^{2}$ and we can impose the following equations

$$
\left\{\begin{array}{l}
\operatorname{Im}\left(f\left(e^{i \beta_{1}^{1} / 2.0}\right)\right)=0 \\
f\left(e^{i\left(\alpha_{1}+\beta_{1}^{1}\right) / 2}\right)=f\left(e^{i\left(\alpha_{1}+\beta_{2}^{1}\right) / 2}\right) \\
\operatorname{Im}\left(f\left(e^{i\left(\beta_{2}^{1}+\beta_{1}^{2}\right) / 2}\right)\right)=0 \\
f\left(e^{i\left(\alpha_{2}+\beta_{1}^{2}\right) / 2}\right)=f\left(e^{i\left(\alpha_{2}+\beta_{2}^{2}\right) / 2}\right) \\
\operatorname{Im}\left(f\left(e^{i\left(\alpha_{3}+\beta_{2}^{2}\right) / 2}\right)\right)=0
\end{array}\right.
$$

Second configuration. We have 8 real unknown parameters: $\operatorname{Re}\left(f^{\prime}(0)\right), \operatorname{Re}\left(b_{1}\right)$, $\operatorname{Re}\left(b_{2}\right), \operatorname{Im}\left(b_{2}\right), \beta_{1}^{1}, \beta_{1}^{2}, \beta_{2}^{2}, \beta_{3}^{2}$ and we can impose the following equations

$$
\left\{\begin{array}{l}
\operatorname{Im}\left(f\left(e^{i \beta_{1}^{1} / 2.0}\right)\right)=0 \\
f\left(e^{i\left(\alpha_{1}+\beta_{1}^{2}\right) / 2}\right)=f\left(e^{i\left(\alpha_{1}+\beta_{2}^{2}\right) / 2}\right) \\
f\left(e^{i\left(\alpha_{2}+\beta_{2}^{2}\right) / 2}\right)=f\left(e^{i\left(\alpha_{2}+\beta_{3}^{2}\right) / 2}\right) \\
\operatorname{Im}\left(f\left(e^{i\left(\alpha_{3}+\beta_{3}^{2}\right) / 2}\right)\right)=0 \\
f\left(e^{i\left(\beta_{1}^{1}+\beta_{1}^{2}\right) / 2}\right)=f\left(e^{i\left(\beta_{3}^{2}+\beta_{2}^{1}\right) / 2}\right)
\end{array}\right.
$$

In Figures 6 and 7 we show an extremal domain for some 6 points for each configuration. As in the last case, these figures represent a conformal map $g$ from the complement of $\mathbb{D}$ onto $\Omega$ such that $g(\infty)=\infty$.

Remark 3. Note that the solution of the problem depend continuously on the parameters $a_{i}$, so if we have one solution for some given set points we can do continuation to reach to any other set of points (with the same topological configuration). This has been used and we did the classic continuation (i.e. for the new set of points we take as a initial condition the solution of the last set of points).

Remark 4. In the implementation of the method, we found a problem when the distance between the arcs on the unit circle is very small, we can't integrate properly the differential equation because we are near the poles $b_{i}$. However this can be overcome by a change of variables. This has been implemented for the special case of the first configuration mentioned in the case of 6 points. In fact, for the 


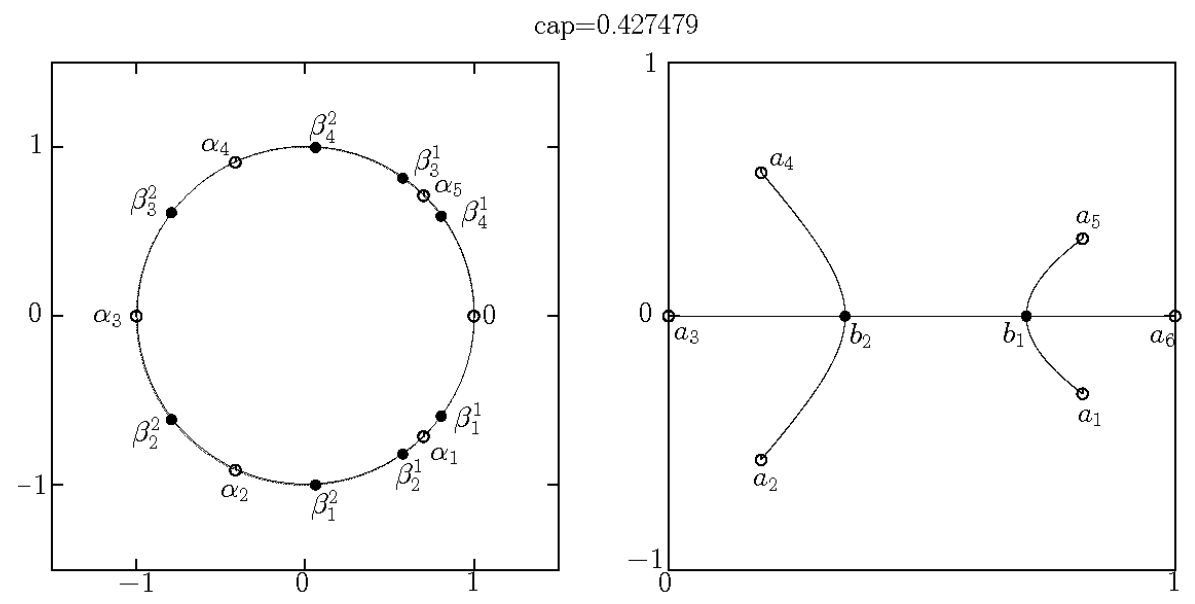

Figure 6. Extremal domain for $n=6$ with symmetry (configuration 1)

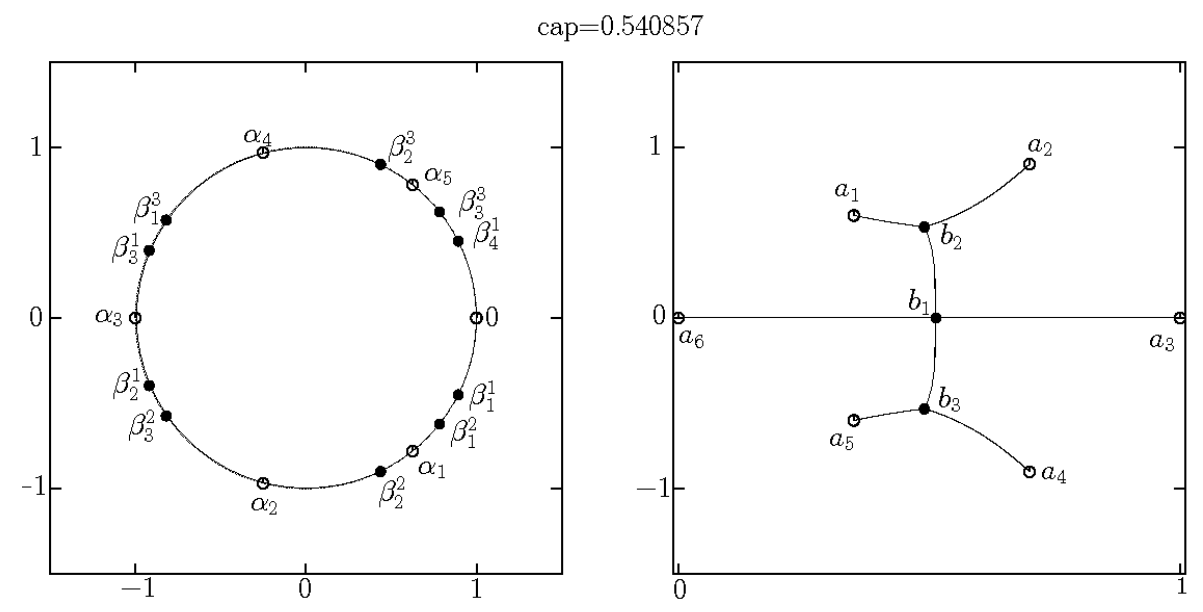

Figure 7. Extremal domain for $n=6$ with symmetry (configuration 2)

application of the Pólya-Chebotarev we only needed the values of $f^{\prime}(0), b_{1}, b_{2}$. This data is enough to obtain the expansion in series of the mapping $f$. This can be done in the following way: given the points $a_{i}$, we have an initial guess for the unknown values. So, we compute the critical orbit starting at point $a_{1}$ till the point with imaginary part equal to $\operatorname{Im}\left(a_{1} / 2.0\right)$ and same for the critical orbit starting at $b_{1}$. The real part of the two points obtained should match if $b_{1}, b_{2}$ are the desired solution. So this is one real equation. Same can be done for the couple of points $a_{2}$ and $b_{2}$. So we have two real equations. The last equation can be $\operatorname{Im}\left(f\left(e^{i 0.1}\right)\right)=0$ (this is valid if the points $a_{1}, a_{2}$ are a bit far from the point $a_{6}$ ). 


\section{Applichtions of the Pólya-Tchebotaröv problem}

The fundamental frequency of a domain. In 1965, Endre Makai (see Mak65) proved the following theorem solving a problem in the study of vibrating membranes raised by Pólya and Szegö in their book [PS51. In 1978, Hayman (Hay78) unaware of it, reproved the same result.

Theorem 4. Let $D$ be a simply connected domain in the complex plane. Let $R_{D}$ be the inradius of $D$, that is, the radius of the largest disc contained in $D$ and let $\lambda_{D}$ be the first Dirichlet eigenvalue for the Laplacian in D. There is a universal constant a such that

$$
\lambda_{D} \geq \frac{a}{R_{D}^{2}} .
$$

There have been many efforts to find the best constant $a$ and to identify the extremal domain for $a$. Makai's proof also shows that the best $a$ satisfies $1 / 4 \leq$ $a<\pi^{2} / 4$.

The following lemma is useful for giving upper bounds for this constant (see [BC94, Lemma 1.2] for a proof):

Lemma 3. Let $J_{0}(r)$ be the first Bessel function and $j_{0}$ the smallest positive zero of $J_{0}$. Assume that $D$ is a simply connected domain. Then

$$
\lambda_{D} \leq j_{0}^{2} \inf _{F}\left\{\frac{1}{\sum_{n=1}^{\infty}\left|a_{n}\right|^{2} \delta_{n}}\right\}
$$

where

$$
\delta_{n}=n^{2} \frac{\int_{0}^{1} J_{0}^{2}\left(j_{0} r\right) r^{2 n-1} d r}{\int_{0}^{1} J_{0}^{2}\left(j_{0} r\right) r d r}
$$

and the infimum is taken over all conformal mappings $F(z)=\sum_{n=0}^{\infty} a_{n} z^{n}$ from the unit disc onto $D$.

In BC94 Bañuelos and Carroll proved that $0.619<a<2.13$ and provided examples of domains which are close to the extremal domain. They did this relating this problem to two other extremal problems:

The expected lifetime of a Brownian motion. Let $B_{t}$ be the Brownian motion in $D$. Let $\tau_{D}=\inf \left\{t>0: B_{t} \notin D\right\}$ be the first exit time of $B_{t}$ from $D$. Let us denote by $E_{z}\left(\tau_{D}\right)$ the expectation of $\tau_{D}$ under the measure of the Brownian starting at the point $z$ in $D$. It is known that there is a universal constant $b$ such that, whenever $D$ is a planar simply connected domain,

$$
\sup _{z \in D} E_{z}\left(\tau_{D}\right) \leq b R_{D}^{2}
$$

As before, we want to know the best value of $b$ and the extremal domain for this last inequality. It is a fact that if $D$ denotes the unit disc of radius $R_{D}$ then $\sup _{z \in D} E_{z}\left(\tau_{D}\right)=R_{D}^{2} / 2$. It is known that $1.584<b<3.228$ (see [BC94]). In order to give an improved lower bound for $b$ it is useful to know the following result (see [BC94, Lemma 1.1.] for further details):

Lemma 4. Suppose that $F(z)=\sum_{n=0}^{\infty} a_{n} z^{n}$ is a conformal mapping from the unit disc onto a simply connected domain $D$ with $F(0)=z_{0}$. Then

$$
E_{z_{0}}\left(\tau_{D}\right)=\frac{1}{2} \sum_{n=1}^{\infty}\left|a_{n}\right|^{2}
$$


The univalent Bloch-Landau constant. If $f$ is an analytic and one to one mapping from the unit disc, then there exists a universal constant $\mathcal{U}$ such that

$$
R_{f(\mathbb{D})} \geq \mathcal{U}\left|f^{\prime}(0)\right| .
$$

This means that the image of the unit disc under any conformal map $f$ contains discs of radius less that $\mathcal{U}\left|f^{\prime}(0)\right|$. Note that from Koebe's $1 / 4$-theorem, we know that $\mathcal{U} \geq 1 / 4$. The best value of $\mathcal{U}$ is known as the univalent or schlicht BlochLandau constant. We can reformulate this problem in terms of the density of the hyperbolic metric. If $f$ is a conformal mapping from the unit disc such that $f(0)=z$ then the density of the hyperbolic metric is $\sigma(z ; D)=1 /\left|f^{\prime}(0)\right|$. So we have the following inequality

$$
\sigma_{D}:=\inf _{z \in D} \sigma(z ; D) \geq \frac{c}{R_{D}} .
$$

where $c:=\mathcal{U}$. From many years there have been efforts to find bounds for $\mathcal{U}=c$. This constant was introduced in 1929 by Landau Lan29, who proved that $\mathcal{U}>$ 0.566. Reich improved this bound in Rei56] $(\mathcal{U}>0.569)$ and Jenkins in Jen61] gave $\mathcal{U}>0.57088$. Many other gave some improved bounds. There are many domains proposed as the candidate for the extremal domain in order to obtain upper bounds for the Bloch-Landau constant. For example, Robinson in Rob35. proved that $\mathcal{U}<0.658$, Goodman in Goo45 that $\mathcal{U}<0.65647$ and in BH85 Beller and Hummel proved that $\mathcal{U}<0.6564155$. Finally in $\mathrm{COC} 08$ this bound has been improved to $\mathcal{U}<0.6563937$. In this last result the resulting domain had all the inner boundary harmonic symmetric with respect to the origin, see Figure 8 We

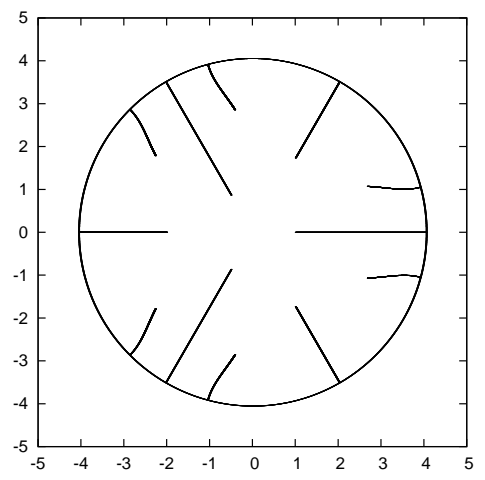

Figure 8. Structure of the domain in $\mathrm{COC} 08$

will use a slight modification of this last domain, which is still harmonic symmetric, to give an improved bound of the Bloch-Landau constant.

As a normalization, we will take domains $D$ with inradius 1 . We will give improved bounds for the constants appearing in the three problems explained above.

Bañuelos and Carroll in BC94 conjectured that the extremal domain is the same for all the three problems. When we restrict these problems to the class of convex domains this is true. In our work, we will see that a similar domain improves the bounds for all the three problems. 
5.1. Bloch-Landau constant. If $h$ is a mapping of the complement of a compact set $E$ onto the complement of the closed unit disc, it can be expanded (up to a rotation) as

$$
h(z)=\frac{z}{\operatorname{cap}(E)}+O(1), z \rightarrow \infty .
$$

So we can relate the problem of the extremal domain for the Bloch-Landau constant with Problem 2 in the case of 6 points because minimizing the capacity is equivalent to increasing the derivative at the origin. It is known (see Car08) that the arcs making up the extremal configuration must be harmonically symmetric at infinity. We will work with domains $\Omega=\Omega_{z_{1}, z_{2}, R}$ as in Figure 9 where $R$ is bigger than 4 and we chose the $\operatorname{arcs} \gamma_{1}, \gamma_{2}, \overline{\gamma_{1}}, \overline{\gamma_{2}}$ so that this domain is harmonically symmetric with respect to 0 . If $g$ is a conformal map of $\mathbb{D}$ onto $\Omega_{z_{1}, z_{2}, R}$ with $g(0)=0$

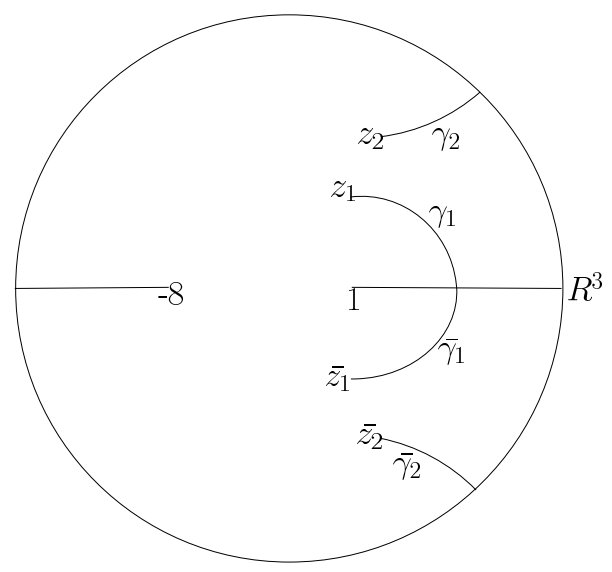

FIGURE 9. Domain $\Omega_{z_{1}, z_{2}, R}$

then $f(z)=z \sqrt[3]{g\left(z^{3}\right) / z^{3}}$ is a conformal map of $\mathbb{D}$ onto the domain $D_{w_{1}, w_{2}, R}$ shown in Figure 10. The arcs in this last domain are harmonically symmetric. We can compute the derivative of $f$ at $0:\left|f^{\prime}(0)\right|=\sqrt[3]{\left|g^{\prime}(0)\right|}$. We need this domain to have inradius 1. Later on we will explain the construction of the domain and the way to get inradius 1. Let $k(z)=z /(1-z)^{2}$ be the Koebe mapping from the unit disc onto the complex plane slit along the negative real axis from minus infinity to $-1 / 4$.

Proposition 1. Let $f$ be the conformal map of $\mathbb{D}$ onto $D_{w_{1}, w_{2}, R}$ such that $f(0)=0$. Then taking $z_{1}=w_{1}^{3}$ and $z_{2}=w_{2}^{3}$,

$$
\frac{1}{\left|f^{\prime}(0)\right|}=\frac{1}{R} \sqrt[3]{|\psi(-8)-\psi(1)| \operatorname{cap}(E)}
$$

where $\psi(z)=-1 / k\left(z / R^{3}\right)$ and $E$ is the continuum with minimal capacity containing 6 given points with symmetry (see Figure 11).

Proof. Using the above notations, consider the map

$$
\phi(z):=\frac{\psi(z)-\psi(1)}{|\psi(-8)-\psi(1)|}
$$




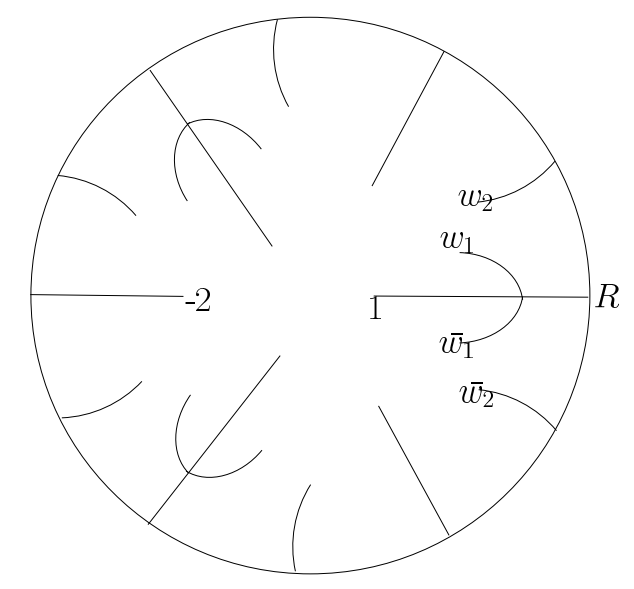

Figure 10. Domain $D_{\omega_{1}, \omega_{2}, R}$

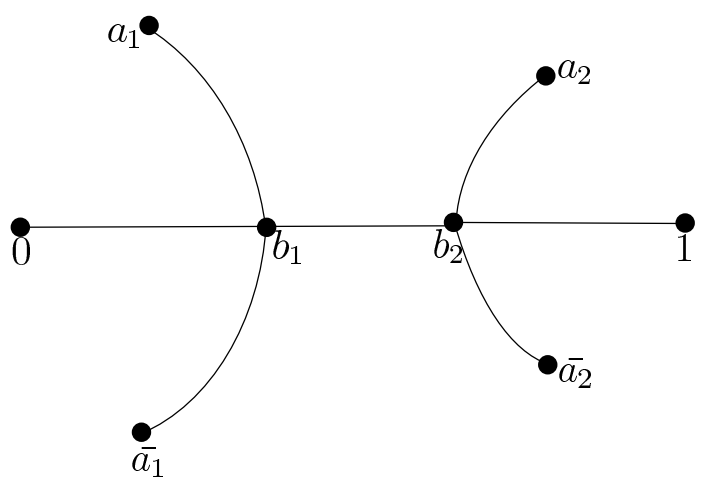

Figure 11. The continuum E

which maps $\Omega_{z_{1}, z_{2}, R}$ onto the complement of the continuum $E$ with $a_{1}=\phi\left(z_{1}\right)$ and $a_{2}=\phi\left(z_{2}\right)(\phi(1)=0, \phi(-8)=1, \phi(0)=\infty)$. Note that the harmonic symmetry of the arcs are preserved since each mapping can be extended continuously to all internal boundary arcs and the domains involved are symmetric with respect to the real axis. Let $h$ be the mapping of the complement of $E$ onto the complement of the unit disc. Then we can define the map $G$ of $\Omega_{z_{1}, z_{2}, R}$ onto $\mathbb{D}$ as

$$
G(z):=\frac{1}{h(\phi(z))} .
$$

We can calculate the derivative $G^{\prime}(0)$ by computing the power series of $G$ :

$$
\left|G^{\prime}(0)\right|=\frac{|\psi(-8)-\psi(1)|}{R^{3} h^{\prime}(\infty)}=\frac{|\psi(-8)-\psi(1)| \operatorname{cap}(E)}{R^{3}} .
$$

The capacity of $E$ can be computed numerically as we explained in 4.2 Now $F:=f^{-1}=z \sqrt[3]{G\left(z^{3}\right) / z^{3}}$ and the proposition is proved. 
5.2. Construction of the domain $D_{w_{1}, w_{2}, R}$. Now we will explain how to obtain the desired domain in order to have inradius 1 and the derivative $\left|f^{\prime}(0)\right|$ as big as possible. In what follows, $D_{p}$ denotes a disc of radius 1 centered at $p$ and $C_{p}=\partial D_{p}$.

The domain is constructed in stages. Let $D(0, R)$ be a disc centered at the origin with radius $R>1$. First we remove from this disc three radial slits that start from the cube roots of the unity. Then, we remove three further slits starting at two times the cube roots of -1 (these are the first two stages of Goodman's domain). Now, as we need inradius 1 we need to put some point in order to not to have discs of radius bigger than 1 . Let $P_{1}=(1+\sqrt{2 \sqrt{3}-3}, 1)$ and denote by $C_{1}$ the circle centered at $P_{1}$ with radius 1 . We have to put some point in $C_{1}$ so that this circle can't increase. Let $w_{1}$ be a point in this circle. Denote by $C_{2}$ the circle of radius 1 tangent to the halfline of argument $\pi / 3$ containing the point $w_{1}$. Let $C_{3}$ be the circle of radius 1, tangent to $|z|=R$ and to the halfline of argument $\pi / 3$ and denote by $w_{2}$ the intersection point of $C_{1}$ and $C_{2}, P_{2}$ and $P_{3}$ the centers of $C_{2}, C_{3}$, respectively (see Figure 12). Now let $\Gamma_{1}$ and $\Gamma_{2}$ be the curves at distance one of

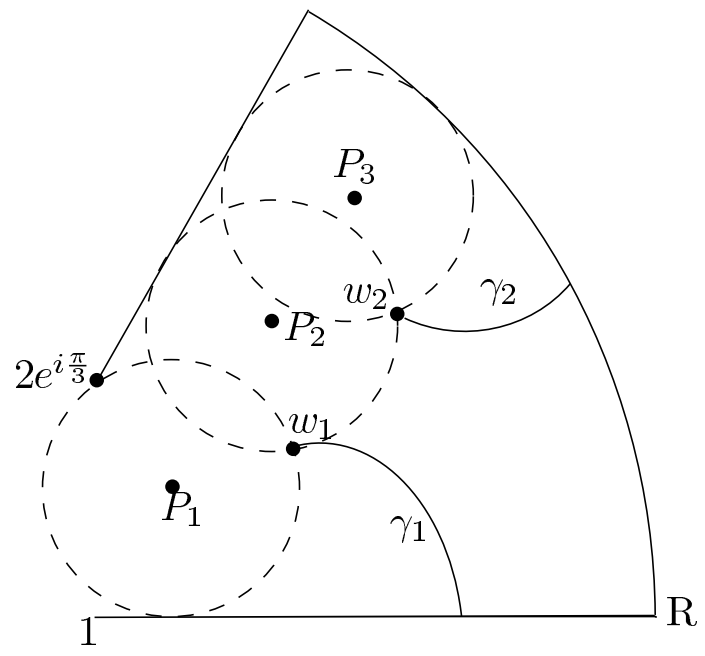

Figure 12. Election of $w_{1}$ and $w_{2}$

$\gamma_{1}$ and $\gamma_{2}$ (i.e. given a point $\gamma_{i}(t) \in \gamma_{i}$, let $v_{n}$ be the normalized orthogonal vector to $\gamma_{i}^{\prime}(t)$, then the corresponding point in the curve $\Gamma_{i}$ is $\Gamma_{i}(t)=\gamma_{i}(t) \pm v_{n}$. Let $q$ denote the intersection of $\Gamma_{1}$ and $\Gamma_{2}$. One sufficient condition to have inradius one is $|q| \geq R-1$. The idea to prove this is to cover all the points $(x, y)$ so that they can't be centers of circles (contained in $D_{w_{1}, w_{2}, R}$ ) with radius bigger than one. Let's show it when our points are located in the sector between the segment $[1, R]$ and the halfline of argument $\pi / 3$. If $z=(x, y)$ is a point such that $y \leq 1,|z| \geq R-1$ or $\operatorname{dist}(z, T) \leq 1$ (where $T$ is the halfline of argument $\pi / 3$ ) then obviously we cant have a circle centered at such point with radius bigger than one. Let $D_{w_{1}}$ and $D_{w_{2}}$ be the discs of radius one centered at the points $w_{1}$ and $w_{2}$, respectively. If $z \in D_{w_{1}} \cup D_{w_{2}}$ then $D_{z}$ contains one of the points $w_{1}$ or $w_{2}$. Therefore, the region $D_{w_{1}} \cup D_{w_{2}}$ is covered. The only risky region is the one between $\partial D_{w_{1}}, \partial D_{w_{2}},|z|=R-1$ and $\{z=(x, y) \mid y=1\}$. But this space is covered by the region delimited by the curves $\Gamma_{1} \cup \Gamma_{2}$ due to the hypothesis (see Figure 13). 


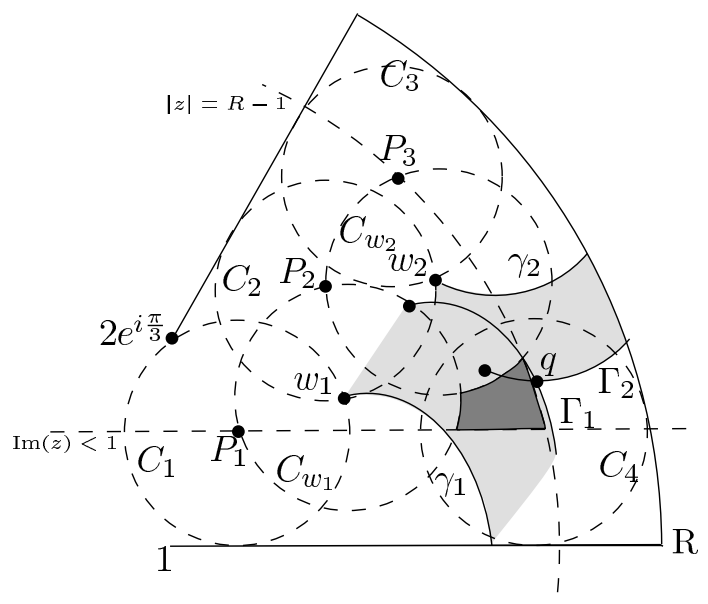

Figure 13. Prohibited zones

5.3. Results. We have computed the bounds for all three problems explained before. To construct the point $w_{1}$ we move on the real axis $x>=1+\sqrt{2 \sqrt{3}-3}$ and define the point $P_{2}$ and then $w_{1}$. After this all gets determined. So given $x$, first we find the biggest $R$ such that $|q| \geq 1-R$, (because the derivative at the origin will increase with the radius $R$ ) and after that we compute the bounds of the constants explained in the three problems. The results obtained are:

(1) For the Bloch-Landau constant, the best upper bound has been found for $x=2.1383799965243$ and $R=5.1195152501$ and the improved bound is

$$
\mathcal{U} \leq 0.656319277272 \text {. }
$$

The domain obtained is shown in Figure 14

(2) Computing the coefficients of the conformal mapping obtained for the domains $D_{w_{1}, w_{2}, R}$, the improved upper bound for the fundamental frequency has been found for $x=2.1282995811037759$ and $R=5.10223601895443$ and it is

$$
a \leq 2.0907934752309 \text {. }
$$

(3) The improved lower bound for the expected life time of a Brownian motion has been found for $x=2.174447128952$ and $R=5.1836816989$ and it is

$$
b \geq 1.670724582110 \text {. }
$$

In all the computations the error estimates that we required are of the order of $10^{-12}$, and we are pretty confident on the correctness of the 10 first digits on the bound, but we have not done a rigorous error analysis.

\section{REFERENCES}

[BA56] A. Beurling and L. Ahlfors, The boundary correspondence under quasiconformal mappings, Acta Math. 96 (1956), 125-142.

[BC94] R. Bañuelos and T. Carroll, Brownian motion and the fundamental frequency of a drum, Duke Math. J. 75 (1994), no. 3, 575-602.

[BH85] E. Beller and J. A. Hummel, On the univalent Bloch constant, Complex Variables Theory Appl. 4 (1985), no. 3, 243-252. 


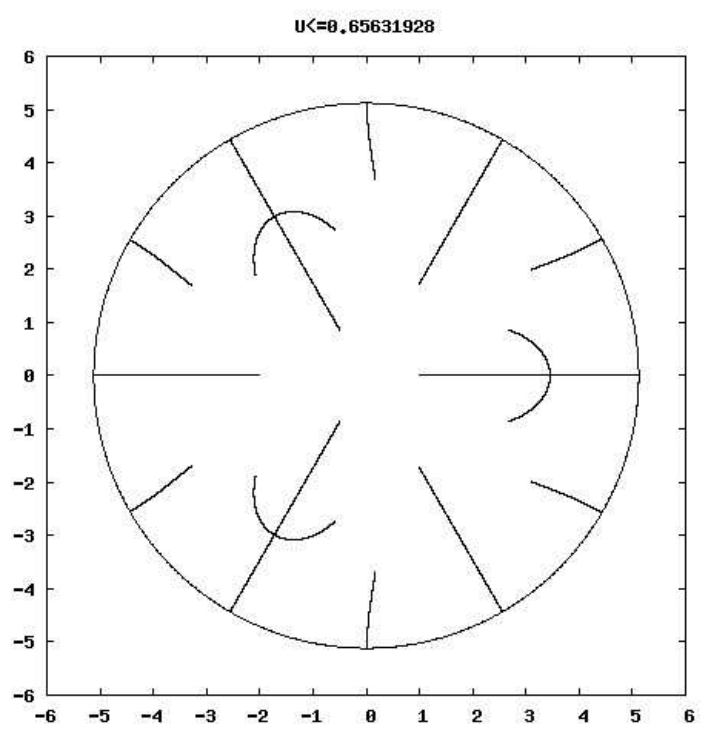

Figure 14. Domain for the improved upper bound of $\mathcal{U}$

[Car08] T. Carroll, An extension of Jenkin's condition for extremal domains associated with the univalent Bloch-Landau constant, Comp. Methods and Function Theory 8 (2008), 159-165.

[COC08] T. Carroll and J. Ortega-Cerdà, The univalent Bloch-Landau constant, harmonic symmetry and conformal glueing, arXiv:0806.2282v1 [math.CV], 2008.

[Cra07] E. Crane, A bound for Smale's mean value conjecture for complex polynomials, Bull. Lond. Math. Soc. 39 (2007), no. 5, 781-791.

[Fed84] S. I. Fedorov, Chebotarev's variational problem in the theory of the capacity of plane sets, and covering theorems for univalent conformal mappings, Mat. Sb. (N.S.) 124(166) (1984), no. 1, 121-139.

[Gol46] G. M. Goluzin, Method of variations in the theory of conform representation, Rec. Math. [Mat. Sbornik] N.S. 19(61) (1946), 203-236.

[Gol69] G. M. Goluzin, Geometric theory of functions of a complex variable, Translations of Mathematical Monographs, Vol. 26, American Mathematical Society, Providence, R.I., 1969.

[Goo45] R. E. Goodman, On the Bloch-Landau constant for schlicht functions, Bull. Amer. Math. Soc. 51 (1945), 234-239.

[Hay78] W. K. Hayman, Some bounds for principal frequency, Applicable Anal. 7 (1977/78), no. $3,247-254$.

[Jen61] J. A. Jenkins, On the schlicht Bloch constant, J. Math. Mech. 10 (1961), 729-734.

[Kuz82] G. V. Kuz'mina, Moduli of families of curves and quadratic differentials, Proc. Steklov Inst. Math. (1982), no. 1, vii+231, A translation of Trudy Mat. Inst. Steklov. 139 (1980).

[Lan29] E. Landau, Über die Blochsche Konstante und zwei verwandte Weltkonstanten, Math. Z. 30 (1929), no. 1, 608-634.

[Lau30] M. Laurentiev, Sur un problème de maximum dans la représentation conforme., C. R. 191 (1930), 827-829 (French).

[Lau34] - On the theory of conformal mappings, Trudy Fiz.-Mat. Inst. Steklov. Otdel. Mat. 5 (1934), 159-245 (Russian).

[Mak65] E. Makai, A lower estimation of the principal frequencies of simply connected membranes, Acta Math. Acad. Sci. Hungar. 16 (1965), 319-323. 
[Pól29] G. Pólya, Beitrag zur Verallgemeinerung des Verzerrungssatzes auf mehrfach zusammenhngende Gebiete. III, Abhandlungen der Preussischen Akademie der Wissenschaften, Physikalisch-Mathematische Klasse (1929), 55-62.

[PS51] G. Pólya and G. Szegö, Isoperimetric Inequalities in Mathematical Physics, Annals of Mathematics Studies, no. 27, Princeton University Press, Princeton, N. J., 1951.

[Pow70] M. J. D. Powell, A hybrid method for nonlinear equations, Numerical methods for nonlinear algebraic equations (Proc. Conf., Univ. Essex, Colchester, 1969), Gordon and Breach, London, 1970, pp. 87-114.

[Rei56] E. Reich, On a Bloch-Landau constant, Proc. Amer. Math. Soc. 7 (1956), 75-76.

[Rob35] R. M. Robinson, The Bloch constant $\mathfrak{A}$ for a schlicht function, Bull. Amer. Math. Soc. 41 (1935), no. 8, 535-540.

[Tam05] P. M. Tamrazov, Tchebotaröv's extremal problem, Cent. Eur. J. Math. 3 (2005), no. 4, 591-605.

Dept. Matemàtica Aplicada i Anàlisi, Universitat de Barcelona, Gran Via 585, 08071 Barcelona, Spain

E-mail address: jortega@ub.edu

Dept. Matemàtica Aplicada i Anàlisi, Universitat de Barcelona, Gran Via 585, 08071 BARCELONA, Spain

E-mail address: bharti@maia.ub.es 